\title{
Chercheurs et communicateurs en communication publique : positionnements dissemblables pour une construction de légitimité disciplinaire en partage?
}

Researchers and communicators in public communication: dissimilar positions for a construction of shared disciplinary legitimacy?

Dominique Bessières, Maître de conférences en sciences de l'information

et de la communication,

Université Rennes 2,

PREFICS (EA 7469) équipe CERSIC dominique.bessieres@univ-rennes2.fr 


\title{
Résumé
}

Cet article propose une réflexion sur les conditions de rapprochements possibles entre chercheurs et communicateurs. Ces acteurs n'obéissent pas aux mêmes attentes, toutefois des «ponts » peuvent être érigés, théoriques et pratiques, entre eux. C'est ce que nous voulons étudier au travers de la communication publique et plus particulièrement de l'association professionnelle du même nom. Avec l'investissement d'un chercheur dans cette association, l'enjeu est de déterminer en quoi la communication publique peut représenter un espace de légitimation en partage pour ces acteurs dissemblables.

Mots clés : communication publique, chercheur, communicateur, intercompréhension.

\begin{abstract}
This article offers a reflection on the possible conditions of rapprochement between researchers and communicators. These actors do not obey the same expectations, however bridges can be builded, theoretically and practically between them. This is what we want to study through public communication and more particularly through the professional association of the same name. With the investment of a researcher in this association, the challenge is to determine how public communication can represent a shared legitimization space for these dissimilar actors.
\end{abstract}

Keywords: public communication, researcher, communicator, intercomprehension. 
La communication publique représente un champ d'action pour les communicateurs ${ }^{1}$, mais aussi pour les chercheurs. Les premiers parce qu'ils ont pour charge la communication des organisations publiques : gestion de prestataires, relations publiques et de presse, événementiel, rédaction de discours, de magazines, de plaquettes, de site web, communication numérique, interface et médiation entre le politique et la structure administrative, conseil en stratégie de communication, voire en gestion d'organisation dans certains cas... Les seconds sont des enseignantschercheurs $^{2}$ qui ont développé tout ou partie de leur recherche à l'étude scientifique de cette communication.

Nous proposons dans le présent article une réflexion sur les conditions de rapprochements entre chercheurs et communicateurs dans la mesure où nous avons pu personnellement enclencher ce type de dynamique. L'enjeu est d'explorer par une approche compréhensive, les manifestations de l'émergence d'une discipline, entendue comme une spécialisation en tant que champ de pratiques sociales des praticiens et comme champ d'étude pour des académiques. Certes les deux champs professionnels n'obéissent pas aux mêmes attentes principielles, toutefois des « ponts » théoriques et pratiques peuvent être érigés entre les deux. Partages d'expériences, de connaissances, sont de nature à former des bases d'une professionnalisation. Notre texte porte sur cet espace de légitimation partagé par les praticiens et les chercheurs, non pas par rapport aux deux groupes d'acteurs dissemblables, mais par rapport à la consolidation d'un champ disciplinaire conjoint, composé de savoirs professionnels et académiques (Bessières, 2009b), d'interactions sociales, de luttes pour imposer des frontières avec d'autres champs sociaux ${ }^{3}$ dont l'accroissement des variétés caractérise les sociétés contemporaines. La communication publique constitue un espace de spécialisation professionnel et un domaine de recherche.

La reconnaissance de la communication publique repose sur des processus d'institutionnalisation de l'activité organisationnelle qui doivent être appréhendés à plusieurs niveaux d'échelle. Il s'agit d'éclairer ces logiques de positionnement. C'est ce qu'il est intéressant d'étudier grâce à l'association professionnelle Communication publique ${ }^{4}$. Principale association de ce secteur en France, fondée en 1989, elle regroupe des directeurs de la communication de l'État, des collectivités locales, des institutions publiques et parapubliques, des établissements publics, des entreprises publiques. Dès lors, en elle-même et par ses actions, elle représente un terrain d'investigation révélateur de logiques sociologiques interactionnistes d'affirmation d'un

1 On trouve aussi les appellations communicant public, directeur ou chargé de communication, dircom...

2 Cf. par exemple : M. Carmes, J.-B. Le Corf, C. Ollivier-Yaniv, I. Pailliart, nous-même...

3 Distinctions par rapport à la communication d'entreprise, à la communication politique...

4 http://www.communication-publique.fr 
champ professionnel. Le travail social de communication institutionnelle publique est une pratique avant d'être un enjeu de définition théorique. C'est ainsi que ce champ social professionnel qu'elle recouvre représente des enjeux disciplinaires pour les communicateurs, mais aussi pour les chercheurs dans la mesure où cette communication peut sembler constituer un "parent pauvre » de la recherche, en comparaison d'autres formes de communication ${ }^{5}$. Nous avons déjà pu montrer dans un article théorique sur la définition de la communication publique (Bessières, 2009a) l'importance des enjeux unificateurs de définition pour des professionnels divers dans leurs profils, leurs périmètres d'action, leurs structures organisationnelles.

Les définitions correspondent à des modèles d'interprétation qui ont des enjeux de représentation. Ceci explique d'abord qu'elles aient été le fait de professionnels (cf. Zémor, 1991 et Le Net, 1993), puis enjeux de luttes entre des disciplines académiques (communication publique pour les sciences de l'information et de la communication (SIC), communication politique pour la science politique (Bessières, 2009a, 2018). Fondamentalement, la définition de cette communication est à relier à son institutionnalisation, en tant que composante des institutions publiques et de leur fonctionnement, comme support de confortement d'un secteur socioprofessionnel et d'une discipline émergente conjuguant des savoirs de praticiens et d'universitaires (Latour, 2007). Elle est produite par divers acteurs : communicateurs publics, enseignants-chercheurs. En effet, la reconnaissance universitaire permet d'acquérir des connaissances académiques (critère de performance) qui ne sont plus seulement empiriques (apprentissage) (Dubar, Tripier et Boussard, 2015). Luc Boltanski (1982) a montré l'importance du développement des formations pour assurer une " production » de nouveaux professionnels nécessaire à la constitution de la dynamique d'un groupe professionnel. Celui-ci peut se faire reconnaitre comme une « profession ${ }^{6}$ » en étant doté progressivement d'attributs, comme des formations diplômantes spécialisées nécessaires avant de pouvoir exercer.

Le concept de discipline renvoie alors conjointement à la désignation de compétences, mais également d'activités scientifiques et de formations. Elle intègre la légitimation par la création de diplômes spécialisés et le développement de recherches au sein des institutions universitaires. L'intérêt porté par des membres des institutions universitaires, par leurs recherches scientifiques et leurs enseignements, contribue à définir le champ des savoirs techniques et conceptuels de la communication publique, en officialisant ses fonctions et ses métiers. Aussi, l'université agit comme une puissante instance de légitimation, de sorte que l'on se rapproche d'une discipline académique, en tant que branche de connaissances faisant l'objet de recherches,

\footnotetext{
5 En sciences de l'information et de la communication, la littérature est par exemple plus conséquente en communication d'entreprise.

6 Nous utilisons le mot profession dans un sens proche de l'anglais « occupation », mais avec des éléments inspirés des professions établies (proche du sens anglais de « profession »).
} 
de publications et de diplômes académiques. Une réserve notable est qu'elle ne correspond pas pleinement à un corps enseignant national spécifique structuré dans les découpages de sections du Conseil National des Universités en France. Mais elle constitue une composante, davantage reconnue aujourd'hui, en particulier dans la $71^{\mathrm{e}}$ section (SIC), dont elle constitue un champ légitime. Néanmoins, il demeure que " cette discipline, encore floue, permet des passages entre les sciences de l'information et de la communication, la science politique, les sciences de gestion par le jeu des qualifications d'une pratique organisationnelle » (Bessières, 2009a). Cette construction disciplinaire contribue aux légitimations de deux catégories d'acteurs : les chercheurs en SIC d'une part, les communicateurs publics d'autre part.

En ce sens, les relations entre chercheurs et praticiens, parfois présentées comme limitées et difficiles (Jeanneret et Ollivier, 2004), ne le sont pas nécessairement dès lors qu'elles sont respectueuses des frontières réelles et symboliques de chaque champ social. Ainsi, en première analyse on peut considérer la frontière entre les champs sociaux de façon restrictive comme un obstacle clôturant (idée de fermeture). Mais en seconde analyse, on peut la concevoir comme une zone de contact, d'ouverture sur l'extérieur (idée de passage). En déclinaison de cette vision, la sociologie fonctionnaliste va souligner la difficulté d'exercice d'un rôle frontalier, tandis qu'il peut exister des possibilités de tenir un tel positionnement sans ressentir de tensions inhibitrices (Bessières et Grima, 1999). En suivant cette seconde acception, la diffusion et le partage des connaissances entre les différents champs d'acteurs peuvent permettre le développement d'une grille partagée de références sur la base d'une altérité réciproque, c'est-à-dire d'une reconnaissance claire des différences de positions et d'identités professionnelles entre les deux groupes d'acteurs (communicateurs et chercheurs).

La matérialité du champ social de la communication publique est objectivée par les actions d'associations professionnelles comme l'association Communication publique, des recherches et des formations diplômantes académiques. Elle concourt à la construction d'une légitimité commune pour ces deux groupes d'acteurs. Pour ce faire, notre approche méthodologique se fonde sur un retour longitudinal visant à appréhender l'institutionnalisation progressive et processuelle de la communication publique en tant que construction disciplinaire. Ceci nécessite une analyse théorique des relations, des cadres et conditions sur lesquels elle repose, rassemblant communicateurs publics et enseignants-chercheurs. Notre projet au cours du texte mobilisera la collecte d'exemples de liens possibles sur plusieurs cas, en prenant notamment appui sur notre propre positionnement frontalier à côté de l'association Communication publique en pointant les différences (première partie), mais également des pistes de rapprochement (seconde partie). 


\section{Interroger la pérennisation de la fonction communication des organisations publiques}

Discerner et analyser des temporalités longues d'institutionnalisation de la communication publique nécessite d'opérer une caractérisation des différenciations des fonctions de communicateurs et de chercheurs puis une recherche de distanciation et d'objectivité afin d'en rendre compte.

\subsection{Distinguer le chercheur du praticien}

Pour étudier la reconnaissance sociale de la communication publique, du groupe professionnel qu'elle recouvre, de son expertise et de sa zone de pouvoir, nous nous attachons aux participations à la production d'identités professionnelles, constituées d'un ensemble de signes ou d'emblèmes qui permettent de regrouper des professionnels (logos, discours, valeurs et modèles proclamés, formations, socialisation). Ainsi entendues, les activités dissemblables de chercheur et de communicateur sont à la source d'identités professionnelles différentes et différenciées, bien que leurs acteurs participent à des processus d'institutionnalisation au profit d'une discipline émergente.

Dans le champ de la communication publique, les responsables de communication institutionnelle gèrent les services de communication des prestataires. Ils définissent plans et actions de communication. Leurs normes et valeurs professionnelles appartiennent à ce que les juristes anglo-saxons dénomment « soft law». À savoir des normes issues de processus d'autorégulation entre pairs dépourvues de la valeur coercitive des textes juridiques (Bessières, 2017). Il en est de même pour les enseignants-chercheurs français, majoritairement fonctionnaires d'État, bénéficiant à ce titre d'une garantie d'indépendance reconnue par les lois de la République ${ }^{7}$, dont les droits et obligations sont codifiés par un large ensemble de normes légales ${ }^{8}$. Ces caractéristiques n'existent pas pour les communicateurs publics, compte tenu de l'importance des contractuels dans ces fonctions en France. Ceci n'offre pas de garanties statutaires équivalentes, en les plaçant dans des situations plus dépendantes des exécutifs (durée d'exercice en tant que contractuels avant de pouvoir bénéficier, sous acceptation des dirigeants publics, d'un contrat à durée indéterminée qui ne permet pas ensuite de progression de carrière (Bessières, 2009b). Toutefois, il faudra mesurer à l'avenir l'application de la Loi de Transformation de la fonction publique du 6 août $2019^{9}$ qui prévoit de nouvelles modalités pour les contractuels au sein des trois fonctions publiques, avec

\footnotetext{
7 Décision du Conseil Constitutionnel n 83-165 du 20 janvier 1984.

8 http://www.enseignementsup-recherche.gouv.fr/pid24530/les-enseignants-chercheurs.html

9 https://www.legifrance.gouv.fr/affichTexte.do?cidTexte=JORFTEXT000038889182\&categorie Lien $=\mathrm{id}$
} 
le principe d'un égal accès aux emplois permanents au travers du «contrat de projet » visant une durée limitée sans que le terme soit précisément connu, ou en fonction des besoins des services. Notamment avec des fonctions nécessitant des compétences spécialisées techniques ou nouvelles, la communication peut facilement rentrer dans ces nouveaux cas de figure.

La reconnaissance dans les champs scientifiques est fonction de la rigueur de la démarche heuristique du chercheur. Elle est évaluée par les pairs dans les publications et communications académiques. Le crédit, la réputation, des chercheurs sont notamment visibles par exemple dans la catégorie administrative " rayonnement » qui correspondent par exemple à des sollicitations d'évaluations de publications, de colloques, d'articles dans des comités scientifiques, ou encore d'expertises de contrats pour des instances de financements de recherche, voire de laboratoire de recherche. C'est un des critères importants et expressément demandé pour des dossiers que les enseignants-chercheurs peuvent être amenés à remplir durant leur carrière pour des promotions, des primes, notamment devant le Conseil National des Universités en France. Les exigences professionnelles des communicateurs (cf. tableau des répertoires ci-dessous) et des chercheurs sont donc structurellement différentes et il ne peut y avoir foncièrement homologie entre ces deux types de professionnalismes, même si on peut trouver des exemples d'enseignants-chercheurs qui ont des attaches, voire des responsabilités dans des associations professionnelles de communicateurs ${ }^{10}$.

\begin{tabular}{|c|c|c|}
\hline $\begin{array}{l}\text { Fonction publique d'État } \\
10 \text { fiches métier }\end{array}$ & $\begin{array}{l}\text { Fonction publique } \\
\text { territoriale } \\
6 \text { fiches métier }\end{array}$ & $\begin{array}{l}\text { Fonction publique } \\
\text { hospitalière } \\
7 \text { fiches métier }\end{array}$ \\
\hline $\begin{array}{l}\text { Directeur de la } \\
\text { communication } \\
\text { Chargé de communication } \\
\text { Chargé de presse } \\
\text { Chargé de la communication } \\
\text { événementielle } \\
\text { Responsable des campagnes } \\
\text { Responsable audiovisuel } \\
\text { Chef de projet multimédia } \\
\text { Chargé de publication } \\
\text { Graphiste } \\
\text { Chargé de promotion et de } \\
\text { diffusion commerciale }\end{array}$ & $\begin{array}{l}\text { Directeur de la } \\
\text { communication } \\
\text { Chargé de communication } \\
\text { Chargé de publication } \\
\text { Photographe-Vidéaste } \\
\text { Chef de projet multimédia } \\
\text { Créateur de support } \\
\text { graphique et audiovisuel }\end{array}$ & $\begin{array}{l}\text { Attaché de presse } \\
\text { Chargé de communication } \\
\text { Cinéaste-Vidéaste } \\
\text { Maquettiste-infographiste } \\
\text { Photographe } \\
\text { Webmestre } \\
\text { Technicien audiovisuel }\end{array}$ \\
\hline
\end{tabular}

Les activités communication des répertoires métiers des 3 fonctions publiques.

Source : Répertoire interministériel des métiers de l'État, des métiers de la fonction publique territoriale, de la fonction publique hospitalière (Bessières, 2009b).

10 Par exemple, dans l'équipe de titulaires du département de Communication de l'Université de Rennes, on dénombre Didier Chauvin pour le réseau Cap'Com, Bruno Chaudet pour l'association Com-Ent, ou encore nous-même pour Communication publique. 


\subsection{Recherche d'impartialité pour un travail de dévoilement}

Dans sa théorisation de la scientificité, Gaston Bachelard (1993) a critiqué le caractère illusoire de l'" expérience première », en introduisant le concept d' " obstacle épistémologique ». Il indique la nécessité de procéder à une rupture entre l'observation et l'analyse. Toutefois, en sciences humaines, nous ne nous situons pas dans une démarche positiviste radicale reposant sur des lois générales, hors de l'expérience, dont la validité ne pourrait être extraite que par un énoncé empiriquement fondé et confirmé. Nous ne nous situons pas davantage dans un constructivisme radical reposant sur des connaissances nécessairement liées à une expérience préalable, individuelle ou collective. La « ...connaissance ce n'est pas "le monde en soi" (le noumène kantien) ou "le monde pour nous" (les phénomènes) ». [...] «L'intérêt des épistémologies constructivistes [permet ainsi d']alimenter et [d']enrichir les problématiques d'analyses des processus d'information et de communication 》(Le Moënne, 2018).

Notre posture de recherche est inspirée, pour partie, par la scientificité théorisée par Bachelard, dans une recherche d'objectivité, au sens d'impartialité, visant à écarter des jugements de valeur, des prénotions, dans la mesure du possible, pour gages de distanciation par rapport à l'objet d'étude, singulièrement avec les valeurs portées par les acteurs sociaux. Il reste que le chercheur doit se départir de la vulgate, des prénotions, du sens commun, des jugements de valeurs des acteurs, pour construire un objet scientifique (Bourdieu, Chamboredon et Passeron, 2005 ; Durkheim, 2013 ; Mucchielli, 2004...). Sur cette base, un travail de dévoilement, constitutif d'une démarche heuristique, peut prospérer. Concrètement, en raison de notre statut d'universitaire, nous n'appartenons pas au groupe professionnel des communicateurs publics. En conséquence, la distanciation est aisée vis-à-vis des enjeux strictement socioprofessionnels du groupe social des communicateurs publics. Ne pas être concerné directement, au premier chef, pour des démarches et processus de recherche, est un facteur propice à l'impartialité, à l'objectivité, à la lucidité. C'est en ce sens que notre démarche peut être vue comme distanciée. Nous ne sommes donc pas en position de ressentir des tensions inhibitrices dans notre action de chercheur.

Dans cette perspective, l'apport du chercheur peut s'avérer essentiel pour analyser les opérations de légitimation du groupe professionnel des communicateurs qui s'inscrivent dans une dimension longue d'une trentaine d'années (l'association Communication publique a été fondée en 1989) mobilisant plusieurs générations d'acteurs de la communication publique. Le travail du chercheur permet d'appréhender les ressorts de l'institutionnalisation de cette communication ; de comprendre les processus longitudinaux, en retraçant en particulier les stratégies de « professionnalisation interactionniste » des acteurs. Ces derniers n'en ont pas globalement forcément toujours pleine conscience. Nous avons abordé ces stratégies en SIC dans des colloques dès 2007 (Bessières, 2018), en termes de dynamisme d'affirmation du groupe professionnel de communicateur vis-à-vis d'autres groupes. 
Depuis lors, la situation a changé et cette voie de recherche semble assez bien représentée, reconnue et intégrée dans les SIC depuis les années 2010 (Brulois et de La Broise, 2010 ; Lépine, 2016 ; Ollivier-Yaniv, 2014...). Le concept de spécialisation des tâches, mis au jour par Émile Durkheim (2007) à la fin du dix-neuvième siècle, identifie ce double mouvement professionnel d'une part, mais également académique d'autre part. C'est un des ressorts des processus complexes d'institutionnalisation de la communication publique.

L'institutionnalisation renvoie intrinsèquement à la perspective de recherche de pérennisation, de consolidation, de formalisation, au moyen d'actions entreprises par des groupes d'acteurs sociaux et peut être définie au travers de trois grandes dimensions (Bessières, 2012) :

- Un processus. La pérennisation de la fonction des communicateurs publics identifie un développement dans la durée. Le programme consiste à faire considérer une telle activité sociale comme allant de soi. C'est l'idée d'une reconnaissance progressive de rôles professionnels sociaux, associée à des valeurs et à des normes mises en lumière par des associations professionnelles notamment. Le temps est incontournable pour y parvenir.

- Une composante des organisations publiques. C'est l'idée d'une intégration de la communication comme une activité «normale » suivant un principe de «naturalisation » de pratiques sociales et organisationnelles. Initiée au départ par la volonté des exécutifs, celle-ci pouvait être contestée, notamment dans les années 1980 avec des critiques tendant à présenter la communication comme une dépense non légitime (cf. les thèmes de la « communication paillette », « la danseuse du président »...). Depuis la fin des années 1990, les actions de communication ne sont plus contestées dans les enquêtes quantitatives réalisées auprès des populations. Mais elles peuvent l'être en tant que dépense pour des raisons conjoncturelles, par des opposants politiques ou en période de restriction budgétaire, parce qu'elles ne sont pas perçues et/ou reconnues par certains acteurs comme un investissement pour mieux faire connaitre les actions auprès des publics destinataires au service d'un objectif de recherche d'efficacité.

- Une « institution sociale » telle que l'a définie Émile Durkheim (2007), à savoir des croyances associées à des modes de conduite. La communication publique correspond effectivement à un ensemble de croyances, révélées notamment par plusieurs auteurs (Miège, 1989 ; Neveu, 2011 ; Sfez, 1988), dans l'efficacité de la communication associées à des exemples pratiques nombreux des organisations privées et publiques (Bessières, 2018), visible dans la généralisation des fonctions de communication dans les champs organisationnels et sociaux.

Dans le secteur public, la relative jeunesse de ces professions et des services fonctionnels transversaux de communication les prive d'une légitimité historique d'antériorité, à la différence des directions opérationnelles sectorielles, comme la 
Direction des ressources humaines (appellation faisant suite à celle plus ancienne de Direction du personnel). Le terrain d'investigation du secteur public est intéressant et révélateur à cet égard, dans la mesure où cette quête de reconnaissance et donc de légitimation est liée notamment au particularisme statutaire de la majorité de ses acteurs (très majoritairement les directeurs et responsables sont contractuels dans des structures fonctionnarisées) (Bessières, 2009b).

Dès lors, la "contribution » du chercheur en SIC à l'émergence de la communication publique en tant que discipline ne peut pas se résumer à une sorte de justification institutionnelle d'une pratique et de ses praticiens. Aussi, s'il est indéniable que certains acteurs sont informés au premier chef des questions qui les animent, ils n'ont pas forcément connaissance d'un cadre théorique et analytique, englobant et surplombant leurs pratiques. Seul un examen longitudinal du champ de la communication publique opéré par un travail autonome de chercheur est de nature à révéler opérationnellement les ajouts successifs aboutissant à la construction collective de la reconnaissance de la fonction communication dans les organisations publiques (Bessières, 2009b). Ce processus constitutif d'une identité professionnelle à visée de légitimation et de cohésion parvient à un « effet de réalité ", celui d'une pérennisation d'un champ professionnel visible dans une généralisation de postes professionnels et une reconnaissance assez récente dans les formations universitaires. À preuve, le ministère de l'Enseignement supérieur a récemment créé une catégorie « communication politique et publique » au cours des années 2010 pour accréditer de nouveaux diplômes de licence et de master en ces matières. C'est là une marque forte de reconnaissance étatique qui contribue, de fait, à labelliser des formations académiques dédiées à des champs professionnels. Mais également, intrinsèquement, elle souligne le rôle académique de soutien des dynamiques de division du travail qui aboutissent à des rôles professionnels plus nombreux et spécialisés, dont l'accès est maintenant plus dépendant d'une formation spécifique. C'est enfin un vecteur essentiel d'institutionnalisation de leur groupe professionnel par son action de régulation (Dubar, Tripier et Boussard, 2015).

Nos travaux s'inscrivent dans le champ de la communication organisationnelle qui semble, depuis les années 2000, reconnaître l'apport des grilles d'analyses interactionnistes, mais également en mobilisant certains apports du constructivisme pour des approches compréhensives, distanciées et critiques (Aldebert et Morillon, 2012).

\section{Observation et intercompréhension}

Le chercheur doit méthodologiquement constituer un terrain de recherche. Des connaissances patiemment tissées peuvent s'avérer importantes pour y parvenir (première partie) et permettre des formes de coopérations (seconde partie). 


\subsection{De l'observation participante à la confiance}

Nous observons, en y participant, depuis plus d'une trentaine d'années des manifestations organisées par des groupements d'acteurs de la communication publique, et particulièrement celles de l'association éponyme ${ }^{11}$ (300 membres, principale association sur ce secteur en France). Elle est l'association professionnelle généraliste la plus importante du champ professionnel ${ }^{12}$. Elle délivre dans ses discours des valeurs, proclamées et proposées pour références identitaires, à destination des membres du réseau professionnel en particulier, mais également vis-à-vis d'autres groupes sociaux, dont les dirigeants publics. En cela, l'association représente un lieu d'échange et de lobbying. Des principes déontologiques sont formalisés (Bessières, 2017) dans cette optique.

La démarche de recherche repose sur des entretiens longitudinaux d'acteurs depuis $1993^{13}$ et sur des observations participantes (Chapoulie, 1984) permettant un accès à des niveaux d'informations difficilement accessibles pour quelqu'un d'extérieur. Notre connaissance de l'association remonte à 1993 au cours de notre thèse de doctorat. Puis notre position de membre de son conseil d'administration nous a permis d'être en mesure de rendre compte de plusieurs actions organisées par l'association professionnelle œuvrant au renforcement du champ professionnel de la communication publique. Il s'agit par exemple d'une action de lobbying visant à inciter les grandes écoles des cadres de services publics à mettre en place des cours de communication publique (Bessières, 2012), d'un référentiel des métiers porté par un collectif d'associations professionnelles, de la communication, d'un manifeste normalisateur du positionnement de l'action et du rôle des communicateurs publics (Bessières, 2016).

Les terrains d'étude constituent alors des supports pour une recherche heuristique par construction et observation. La démarche recherche une neutralité et une distanciation par rapport aux valeurs et discours des acteurs. Tout en gardant à l'esprit que les matières « sociales », empêchent que l'on puisse prétendre à un positivisme à l'instar de certaines sciences « dures » qui produisent des connaissances fondées sur des phénomènes naturels, ou à un empirisme strict rarement atteignable en sciences humaines. Une dimension constructiviste, non radicale, passe par la confrontation aux formes de savoir autres que celles que détient le chercheur (Mucchielli, 2004). Autrement dit, le terrain constitue un accès à un point de vue

\footnotetext{
11 http://www.communication-publique.fr/

12 Des associations plus restreintes pour la communication locale pourraient être citées (Fourdin, 1994).
}

13 Nous ne rentrerons donc pas dans le détail de nos champs de recherche et nous renverrons à nos publications pour cela, dans un ordre de grandeur d'une centaine d'entretiens, auxquels s'ajoutent des contacts et discussions plus informels. 
du scientifique. Le chercheur dans une recherche d'impartialité, de lucidité, par son analyse théorique, éclaire les stratégies de professionnalisation des acteurs, la centralité de la communication publique dans la gestion des organisations (objectifs de modernisation). Notre démarche compréhensive vise à appréhender les ressorts des évolutions notamment par le recueil de discours d'acteurs au travers d'entretiens longitudinaux depuis les années $1990^{14}$. Description et explication permettent de rendre compte du terrain et d'analyser (voire de co-construire lors de participations à des actions dans certains cas) avec ce terrain des propositions de recherche et des résultats. Il s'agit ainsi de considérer le terrain comme un cadre interprétatif permettant d'articuler des connexions entre les idées théoriques dérivées de l'induction et une théorisation scientifique globale publiée.

Le terrain de la recherche doit être pertinent au regard de l'objet de recherche, mais encore faut-il que celui-ci soit accessible! Cette accessibilité peut être conditionnée par une relation de confiance, voire d'intérêt. Cette relation peut-elle être maintenue durant le temps de la recherche (parfois sur plusieurs mois ou années comme une thèse) ? Le terrain permet-il au chercheur un bon niveau d'analyse pour valider ses hypothèses ou bien construire ses propositions ? Ces conditions peuvent s'avérer cruciales pour que le chercheur soit en position de collecter des informations dont il a besoin auprès des bonnes personnes. C'est ce que nous avons pu organiser sur une longue durée avec l'association professionnelle Communication publique.

L'observation participante ouvre un accès privilégié à des exemples d'opérations de groupes sociaux ou d'associations professionnelles qui permettent de comprendre plus finement les dynamiques interactionnistes visant à consolider un champ professionnel. Elle est également de nature à favoriser un dialogue sur des bases incontestables entre les groupes d'acteurs aussi différents que les chercheurs et les communicateurs, tout en pouvant ouvrir la possibilité de confiance réciproque (versus risques d'instrumentalisation du chercheur $^{15}$ ). Pour le chercheur, l'observation participante (Chapoulie, 1984 ; Mucchielli, 2004) permet l'accès à des terrains privilégiés, ce qui peut s'avérer intéressant et fructueux pour recenser des pratiques, les expliciter et en rendre compte scientifiquement.

14 Cf. des entretiens de directeurs et chargés de la communication en régions Île de France, Champagne-Ardenne, de ministères, de collectivités territoriales, de CCI, d'associations professionnelles, de conseils en communication, de journalistes spécialisés entre 1993 et 2019, d'une durée de 40 minutes pour les plus brefs à 2 heures et plus pour certains.

15 Nous n'avons par exemple jamais été confronté à des demandes des membres de l'association pouvant entraver nos fonctions d'enseignant-chercheur. 


\subsection{Intercompréhension des connaissances et diplomation}

Au niveau du travail de production de connaissances scientifiques, il est possible de construire des artéfacts théoriques, « hors sol», et sans grande étendue explicative par rapport à un monde professionnel précis. Le risque serait de ne pas avoir une grande portée et d'être finalement peu pertinent tant auprès des acteurs de terrain que de la communauté scientifique. Plus largement la construction d'un objet de recherche mobilise des lectures théoriques, mais aussi des confrontations avec des terrains, singulièrement auprès de professionnels et de leurs préoccupations. Un tel processus constitue une assurance d'une plus grande pertinence en fonction d'une intelligence du contexte qui peut alimenter et influencer la démarche de construction de l'objet scientifique.

Cet état de fait ne constitue pas pour autant une limite pour le chercheur, dès lors que demeure une démarche de recherche d'objectivité scientifique, éloignée de préoccupations polémistes, comprise comme telle par les acteurs sociaux observés. Une dimension critique, dès lors qu'elle est exprimée avec une neutralité formelle dans un souci d'objectivité scientifique, n'est pas de nature à soulever des oppositions, des contestations, des conflits avec les acteurs sociaux observés. Ceci d'autant plus que les communicateurs publics peuvent être plus questionnés par des problématiques d'évaluation dans une visée managériale et de contrôle des coûts dans des contextes budgétaires globalement plus contraints (Bessières, 2010). En outre, ceux-ci peuvent trouver un intérêt intellectuel aux écrits et productions scientifiques, a fortiori s'ils les concernent.

Éprouver des théories et des concepts, les mettre en tension avec des savoirs pratiques ou pour expérimenter, à tout le moins afin de s'assurer de ne pas faire de contresens sur l'interprétation des réalités observées, permet de penser et conceptualiser des constructions théoriques sur la base de ces informations initiales. Le chercheur doit souvent confronter ses études qualitatives, dont ses entretiens, avec le terrain dans sa collecte de données. Il doit introduire une perspective d'analyse scientifique qui sera validée par ses pairs, ce qui permet d'éviter qu'un seul point de vue, celui d'un seul groupe ou d'une seule personne, ne soit privilégié.

Le risque pour le chercheur est celui d'interprétations hasardeuses, de projections dont nous sommes prémunis sur le terrain de la communication publique par une bonne connaissance directe des enjeux et thématiques portés les professionnels. La position frontalière représente un atout non négligeable. Lorsque notre connaissance était moindre, nous avons par exemple compensé en proposant une coécriture à un praticien-chercheur immergé dans ce secteur, avec qui nous avons pu mener une enquête sur le milieu très spécifique de la Police Judiciaire de la Jeunesse, au sujet du rôle communicationnel des écrits professionnels (Bessières et Matuszak, 2016). Ceci a permis de valider notre grille d'analyse théorique, tout en l'enrichissant de sa parfaite connaissance du vocabulaire et de l'ampleur des normes juridiques pesant sur 
ce secteur du Ministère de la Justice. L'intention était d'éviter des risques de sur ou de sous interprétation tout en cherchant à économiser du temps pour tenir les délais d'un contrat de recherche de notre part, de prendre davantage de distance critique et théorique de la part de notre co-écrivant par l'entremise d'un double regard.

L'intercompréhension et la coopération sont également présentes dans notre responsabilité du Prix du meilleur mémoire de communication publique porté par l'association Communication publique, en partenariat depuis 2014 avec l'Association des Responsables de Communication de l'Enseignement Supérieur (ARCES) et la Société Française des Sciences de l'Information et de la Communication (SFSIC). Ces partenariats accentuent et renforcent la double vocation professionnelle et universitaire de ce type de travail. Le jury est appelé à suivre des consignes que nous avons élaborées pour cadrer et harmoniser les modes d'évaluation, accompagnées d'un modèle de fiche de notation. Ce pont entre les champs des communicateurs professionnels et des enseignants-chercheurs permet l'analyse des constructions et conceptualisations d'étudiants de masters 2 nationaux et internationaux francophones. Plus largement, nous avons pu montrer (Bessières, 2009a) que les prix professionnels contribuent à forger une représentation de l'actualité de la communication publique au travers d'exemplarités récompensées. C'est donc en partie à une échelle microsociale (prix et salons, déontologies, associations...) que s'articulent certains ressorts du renforcement d'un groupe professionnel.

L'intercompréhension peut être obtenue également dans des rapprochements dans une revue professionnelle mobilisant des regards croisés. Par exemple Parole publique. La revue de la Communication publique qui existe depuis mars 2013 est ouverte au monde de la recherche universitaire et nous sommes d'ailleurs membre de son comité de rédaction. Les articles publiés sont produits par des praticiens, des dirigeants publics, mais avec la volonté de proposer une place importante aux écrits de chercheurs. Ceux-ci proviennent de divers horizons académiques, systématiquement présents et éclairants des problématiques de fond concernant les contextes d'exercice de la communication publique.

La légitimation d'une discipline, en tant que conjonction de savoirs professionnels issus des pratiques et de savoirs universitaires, incline à des relations d'interactions entre chercheurs et communicateurs. Les chercheurs, en participant à des associations professionnelles, peuvent œuvrer au développement de la reconnaissance des métiers de communicateurs. De même, cette présence du chercheur dans les associations professionnelles peut participer à la reconnaissance de diplômes ou d'enseignements professionnalisants. Plus largement, les exigences de professionnalisation de la communication sont à l'origine des SIC (Boure, 2006). Elles sont donc concernées par des demandes sociales, comme le souci d'entretenir de bonnes relations avec les « champs professionnels » afin de faciliter une meilleure entrée sur le marché du travail des diplômés. 


\section{Conclusion}

Pour un chercheur, être au carrefour d'un champ professionnel, comme celui de la communication publique, est une position frontalière qui non seulement peut ne pas être difficile, mais qui peut représenter une clé d'accès à des terrains d'observation et de recherche de façon plus aisée. La diffusion et le partage des connaissances entre les différents champs d'acteurs peuvent a fortiori s'avérer déterminants dans le développement d'une grille commune de références sur la base d'une altérité réciproque.

L'enjeu est aussi celui de la légitimation d'une activité - la communication publique - dont le chercheur peut être acteur par son travail de recherche. Sa position et son mode de raisonnement peuvent contribuer à accroître la rationalité des acteurs qu'il observe et avec qui il a intérêt à entretenir des relations de confiance. Audelà de la lecture de ses écrits par les communicateurs, de manière réciproque le chercheur s'intéresse à leurs actions, écrits et manifestations. Ceci est de nature à permettre le développement de cette grille commune de références, réalisant ainsi l'intercompréhension et le partage de connaissances. Aujourd'hui, ce qu'il y a peutêtre de nouveau, semble résider dans l'importance de la communication comme facteur global de professionnalisation. On mesure l'émergence d'une discipline, entendue comme spécialisation d'un champ socioprofessionnel de pratiques sociales des praticiens et d'un champ d'études émergent pour des académiques. S'y ajoutent des enjeux de diplomation et donc de pérennisation d'un groupe professionnel spécifique. Le chercheur par son analyse théorique contribue à éclairer et à dévoiler les enjeux sociologiques et communicationnels des articulations plus importantes qui tendent à devenir structurelles avec le temps, entre des champs pourtant fortement dissemblables. L'enjeu réside dans le partage d'une légitimité au service d'une continuité d'existence de la communication publique.

\section{Bibliographie}

Aldebert B. et Morillon L. (2012). Communication des organisations : comparaison des approches scientifiques en gestion et en communication. RIHM, 13(2) (numéro spécial), 59-77. http://europia.org/RIHM/V13N2/4-RIHM13(2)-Aldebert.pdf

Bachelard G. (1993). La Formation de l'esprit scientifique (1938). Paris, France : Vrin.

Bessières D. (2009a). La définition de la communication publique : des enjeux disciplinaires aux changements de paradigmes organisationnels. Communication \& Organisation, 35, 15-28.

doi : https://doi.org/10.4000/communicationorganisation.686 
Bessières D. (2009b). La quête de professionnalisation des communicateurs publics entre difficulté et stratégie. Formation Emploi, 108, 39-52. http://journals.openedition.org/formationemploi/2081

Bessières D. (2010). L'évaluation de la communication publique, entre norme gestionnaire et légitimités, des enjeux difficilement conciliables ? Communication \& Organisation, 38, 63-74. doi : https://doi.org/10.4000/communicationorganisation.1407

Bessières D. (2012). L'institutionnalisation des communicateurs publics : une tension longitudinale vers la professionnalisation pour une légitimation du groupe professionnel. Pyramides, 24(2), ULB (Belgique), 239-260.

URL : http://journals.openedition.org/pyramides/953

Bessières D. (2016). Communication publique et association professionnelle : légitimation et normalisation. Revue française des sciences de l'information et de la communication, 9. doi : https://doi.org/10.4000/rfsic.2003

Bessières D. (2017). Les régulations professionnelles déontologiques de communication publique : des valeurs et des normes de professionnalisation vecteurs de reconnaissance. Communication \& Professionnalisation, 5, 54-72. doi : https://doi.org/10.14428/rcompro.vi5.873

Bessières D. (2018). La communication publique, Chapitre 22. Dans T. Libaert (dir.), Communication (pp. 201-227). Paris, France : Vuibert.

Bessières D. et Grima F. (1999). Quelles légitimités pour les services fonctionnels de la communication et de la formation dans les collectivités locales. Politiques et Management Public, 4, 1-16. doi : https://doi.org/10.3406/pomap.1999.2250

Bessières D. et Matuszak C. (2016). La communication autour des rapports des éducateurs destinés aux magistrats : attentes et évaluations croisées. Dans P. Delcambre et C. Matuszak (dir.), Écrire au magistrat. Nouvelles normes, nouvelles contraintes (pp. 79-104). Villeneuve d'Ascq, Presses universitaires du Septentrion.

Boltanski L. (1982). Les Cadres, la formation d'un rôle social. Paris, France : Les Éditions de Minuit.

Bourdieu P., Chamboredon J.-C. et Passeron J.-C. (2005). Le Métier de sociologue. Préalables épistémologiques, $5^{\mathrm{e}}$ éd. Paris, France : EHESS.

Boure R. (2006). L'histoire des sciences de l'information et de la communication. Entre gratuité et réflexivité. Questions de Communication, 10, 277-295. doi : https://doi.org/10.4000/questionsdecommunication.7718 
Brulois V. et de la Broise P. (2010). La communication interne aux prises avec la professionnalisation. Communiquer, 3-4, 123-134. doi : https://doi.org/10.4000/communiquer.1584

Chapoulie J.-M. (1984). Everett C. Hughes et le développement du travail de terrain en sociologie. Revue française de sociologie, 25(4), 582-608.

Dubar C., Tripier P. et Boussard V. (2015). Sociologie des professions, $4^{e}$ éd. Paris, France : Armand Colin.

Durkheim É. (2007). De la division du travail social (1893). Coll. Quadrige. Paris, France : Presses universitaires de France.

Durkheim É. (2013), Les Règles de la méthode sociologique, 14éd., Paris, France : Presses universitaires de France.

Fourdin M (1994). La professionnalisation de la communication locale : un paradoxe?, Réseaux, 64(12), mars-avril, 75-89. doi : https://doi.org/10.3406/reso.1994.2470

Jeanneret Y. et Ollivier B. (2004). Faire des SIC : praxis, méthodes, pratiques. Hermès, 38, 130-132. doi : https://doi.org/10.4267/2042/9437

Latour B. (2007). Petites leçons de sociologie des sciences. Paris, France : La Découverte poche.

Le Moënne C. (2018). Penser l'artificialisation du monde ? Retour sur la question du constructivisme et la transformation du numérique, Communication \& Organisation, 53,107-132.doi:https://doi.org/10.4000/communicationorganisation.6160

Le Net M. (1993). Communication publique-Pratiques des campagnes d'Information. Paris, France : La Documentation française.

Lépine V. (2016). Penser la professionnalisation comme une mise en mouvement : les communicateurs. Revue Française des Sciences de l'information et de la communication, 9. doi : https://doi.org/10.4000/rfsic.2266

Miège B. (1989). La Société conquise par la communication. Grenoble, France : Presses universitaires de Grenoble.

Mucchielli A. (dir.) (2004). La Place du constructivisme pour l'étude des communications, Actes du colloque de Béziers, 2003. Montpellier, France : Presses universitaires de la Méditerranée.

Neveu E. (2011). Une société de communication? Paris, France : Montchrestien.

Ollivier-Yaniv C. (2014). La communication publique comme «monde social» : une approche réflexive. Dans P. Aldrin, N. Hubé, C. Ollivier-Yaniv et J.-M. Utard (dir.), Les Mondes de la communication publique. Légitimation et fabrique symbolique du politique. Rennes, France : Presses universitaires de Rennes. 
Sfez L. (1988). Critique de la communication. Paris, France : Seuil.

Zémor P. (2008). La communication publique (1 $1^{\text {re }}$ édition 1991). Coll. Que sais-je, $\mathrm{n}^{\circ} 2940$ (pp. 419-423). Paris, France : PUF. 